\title{
Study of the effect of Buteyko breathing technique in patients with hypertension - A case series
}

\author{
Visalakshi H Subramanian ${ }^{1 *}$, Rachna D Arora ${ }^{2}$ \\ ${ }^{1}$ Lecturer, ${ }^{2}$ Assistant Professor, Dept. of Physiotherapy, Dr. B R Ambedkar Medical College and Hospital, Bangalore, Karnataka, ${ }^{2} \mathrm{PT}$ \\ School \& Center, Topiwala National Medical College \& B Y L Nair Ch Hospital, Mumbai, Maharashtra, India
}

*Corresponding Author: Visalakshi H Subramanian

Email: dr.physio.vhs@outlook.com

\begin{abstract}
Introduction: The condition in which the blood vessels have persistently raised pressure is called as hypertension. Dr. K P Buteyko developed a set of breathing exercises called the Buteyko Method. The breathing techniques of this method are unusual in that people are asked to breathe less rather than more and holding the breath is considered as important as breathing. The breathing here is a nasal breathing pattern.
\end{abstract}

Aim: To assess the haemodynamic changes following Buteyko Breathing Technique training for 2 weeks in hypertensive subjects

Objectives: To assess the effects of the Buteyko Breathing Technique on pulse rate, blood pressure and control pause by.

Materials and Methods: After screening for inclusion and exclusion criteria, 6 participants were included in the study.

The intervention duration was for 2 weeks, 6 days/week and the number of sessions were recorded. The technique was taught through a DVD. Each session lasted for 15 - 20 minutes. Sessions were conducted at a fixed time every day.

Results: The mean reduction in pulse rate was $2.5 \pm 1.04 /$ minute, systolic blood pressure $4.16 \pm 3.76 \mathrm{~mm}$ of $\mathrm{Hg}$ and diastolic blood pressure $5 \pm 3.16 \mathrm{~mm}$ of $\mathrm{Hg}$ respectively.

Conclusion: There was a reduction in pulse and BP following 2 weeks of Buteyko training.

Keywords: Hypertension, Buteyko breathing technique, Breath-holding techniques, Control, Pause, Carbon dioxide retention.

\section{Introduction}

Approximately 17 million deaths in a year occur due to cardiovascular disease globally. Every year, hypertension and its complications are accountable for 9.4 million deaths worldwide. ${ }^{1}$ In south Asia, Hypertension (HTN) is ranked as the third important risk factor that is attributable to the burden of disease. ${ }^{2}$ In India, HTN is directly attributable for $57 \%$ of all deaths due to stroke and $24 \%$ of all deaths due to coronary heart disease (CHD). ${ }^{3,4}$

Dr K P Buteyko developed a set of breathing exercises called The Buteyko Method. The aim was to develop breathing patterns that helps to maintain the correct ratio of oxygen and carbon dioxide in the bloodstream, in a healthy way. The breathing techniques of the Buteyko Method are unusual because a person is asked to breathe less rather than more, and not breathing or holding the breath is considered as important as breathing. ${ }^{5}$

The Buteyko Method incorporates reduced-volume breathing as its fundamental technique, which is done by sitting in an erect posture and relaxing the muscles of respiration until one feels a slight lack of air. This sensation of slight breathlessness is then maintained by a combination of relaxation of breathing muscles, erect posture, and a little tension of the abdomen. In a formal practice session, combination of breath-holding techniques is used to perform the reduced-volume breathing, the two most important being a short breath hold, called the Control Pause, and a longer breath hold, called the Maximum Pause. The breathing here is a nasal breathing pattern.

\section{Need for the study}

Buteyko breathing technique or the Buteyko method is an alternative to breathing technique used for in conditions like asthma and other obstructive airway disease. This method emphasises on the breathing pattern which corrects the hyperventilation (deeper breathing rate). At the core it focuses on breathing through the nose, that is, the Nasal breathing, Breath Holding and relaxation. ${ }^{6}$

The Buteyko breathing technique emphasises on the optimal carbon dioxide retention which in turn has many beneficial effects. The carbon dioxide has a vasodilatory effect, because of which there is a reduction in the pulse rate. Thus, this study attempts to find the effect of Buteyko breathing technique in hypertensive patients.

\section{Aim and Objectives \\ Aim}

To assess haemodynamic changes following 2 weeks of Buteyko breathing in subjects with Hypertension.

\section{Materials and Methods}

The study is a case series with 6 hypertensive subjects. The subjects who were diagnosed with Hypertension and were on anti-hypertensive drugs for at least 1 year and willing to participate were included in the study, whereas, the subjects who had been hospitalised in the past one year due to cardiac disease were excluded from the study. Outcomes measured were pulse rate, blood pressure and control pause. The materials used in the study were sphygmomanometer, stethoscope, stopwatch, pulse oxymeter, laptop and DVD on Buteyko Breathing Technique. 
Table 1: Differences in means, SEM and MDC for pulse, blood pressure and control pause in a case series study ( $\mathrm{n}=6)$

\begin{tabular}{|l|c|c|c|c|}
\hline & $\begin{array}{c}\text { Differences in } \\
\text { means }\end{array}$ & Remarks & $\begin{array}{c}\text { Standard error } \\
\text { of measurement }\end{array}$ & $\begin{array}{c}\text { Minimal detectable } \\
\text { change value }\end{array}$ \\
\hline Pulse rate / minute & $2.5 \pm 1.04$ & Reduction & 0.42 & 1.18 \\
\hline Systolic blood pressure (mm of Hg) & $4.16 \pm 3.76$ & Reduction & 1.53 & 4.25 \\
\hline Diastolic blood pressure & $5 \pm 3.16$ & Reduction & 1.29 & 3.57 \\
\hline MEANCP & $5 \pm 0.89$ & Increase & 0.36 & 1.01 \\
\hline
\end{tabular}

\section{Procedure}

After screening for inclusion and exclusion criteria, participants were included in the study. Written consent was taken after the study procedure was explained. The information pertaining to demographic details, vital parameters, anthropometric measures, co-morbidities and current medication were recorded.

The blood pressure, pulse rate and control pause were recorded at baseline and at the end of 2 weeks. The intervention was 6 days per week. The Technique was taught through a DVD. Each session lasted for $15-20 \mathrm{~min}$, where the time of the day was fixed for the sessions.

\section{Results}

The differences in measures, pre and post intervention were mean reduction in pulse rate $2.5 \pm 1.04$ /minute, systolic blood pressure $4.16 \pm 3.76 \mathrm{~mm}$ of $\mathrm{Hg}$ and diastolic blood pressure $5 \pm 3.16 \mathrm{~mm}$ of $\mathrm{Hg}$ respectively (Table 1). The mean increase in control pause was $5 \pm 0.89$ (range 4 to 6 ) seconds.

\section{Discussion}

The mean difference in pulse rate (2.5) was higher than the Minimal Detectable Change (1.18). The mean difference in systolic blood pressure (4.16) was lower than or similar to the Minimal Detectable Change (4.25) for their difference being very minimal (0.09). The mean difference in diastolic blood pressure (5) was higher than the Minimal Detectable Change (3.57). The mean difference in Control Pause (5) was higher than the Minimal Detectable Change (1.01). This suggests that the Buteyko Breathing technique had a significant effect on diastolic blood pressure, pulse rate and control pause.

This can be attributed to the vasodilation due to the carbon dioxide retention, that further leads to the lowering of peripheral resistance and hence the reduction in diastolic blood pressure. ${ }^{5}$

A review published in 2003 stated that, $5 \mathrm{mmHg}$ reduction in blood pressure can decrease the risk of stroke by $34 \%$, of ischeamic heart disease by $21 \%$, and decrease the likelihood of heart failure, dementia and mortality from cardiovascular disease. (Law et al. $)^{8}$

\section{Conclusion}

There was a reduction in pulse and diastolic blood pressure and an increase in Control Pause duration in Hypertensive patients following 2 weeks of Buteyko training.

\section{Acknowledgement}

We sincerely thank Mrs. Chhaya. V. Verma, Professor and Head, P.T. School \& Center, Topiwala National Medical College and B.Y.L Nair Ch Hospital, Mumbai for guiding us from the conception of the study. We extend our heartfelt thanks towards the participants of this study.

\section{Funding}

Not applicable

\section{Conflict of interest}

No conflict of interest is declared by the authors.

\section{References}

1. WHO A. global brief on hypertension. Silent killer, global public health crisis. World Health Organization, Geneva, Switzerland. 2013 Sep 10.

2. Lim SS, Vos T, Flaxman AD, Danaei G, Shibuya K, AdairRohani H, AlMazroa MA, Amann M, Anderson HR, Andrews KG, Aryee M. A comparative risk assessment of burden of disease and injury attributable to 67 risk factors and risk factor clusters in 21 regions, 1990-2010: a systematic analysis for the Global Burden of Disease Study 2010. Lancet 2012;380(9859):2224-60.

3. Reddy KS, Shah B, Varghese C, Ramadoss A. Responding to the threat of chronic diseases in India. Lancet 2005;366(9498):1744-9.

4. Leeder S, Raymond S, Greenberg H, Liu H, Esson K. A race against time: the challenge of cardiovascular disease in developing economies. New York: Columbia University. 2004 Apr 25.

5. Rosalba Courtney Strength, weakness, possibilities of Buteyko Breathing Method, Association for Applied Psychophysiology \& Biofeedback; 2008;32(2):59-63.

6. Buteyko factsheet: Asthma and Respiratory Foundation of New Zealand 2010.

7. Dontje ML, Dall PM, Skelton DA, Gill JM, Chastin SF, Seniors USP Team. Reliability, minimal detectable change and responsiveness to change: Indicators to select the best method to measure sedentary behaviour in older adults in different study designs. PloS One. 2018;13(4):e0195424.

8. Law M, Wald N, Morris J. Lowering blood pressure to prevent myocardial infarction and stroke: a new preventive strategy. In NIHR Health Technology Assessment programme: Executive Summaries 2003. NIHR Journals Library.

How to cite this article: Subramanian VH, Arora RD. Study of the effect of Buteyko breathing technique in patients with hypertension - A case series. J Soc Indian Physiother 2020;4(1):14-5. 